\title{
Spectroscopic investigations of classical Cepheids and main-sequence stars in galactic open clusters and associations
}

\section{Association Cas OB2 and the small-amplitude Cepheid SU Cassiopeae}

\author{
I. A. Usenko ${ }^{1,3}$, V. V. Kovtyukh ${ }^{1,3}$, V. G. Klochkova, ${ }^{2,4}$, V. E. Panchuk ${ }^{2,4}$, and S. V. Yermakov ${ }^{2}$ \\ 1 Astronomical Observatory of Odessa State University, Odessa 65014, Ukraine \\ e-mail: val@deneb.odessa.ua \\ 2 Special Astrophysical Observatory, Russian Academy of Sciences, Nizhny Arkhyz, Stavropol Territory, 369167, \\ Russia \\ e-mail: valenta@sao.ru; panchuk@sao.ru; ermak@sao.ru \\ 3 Isaac Newton Institute of Chile, Odessa Branch \\ ${ }^{4}$ Isaac Newton Institute of Chile, SAO RAS Branch
}

Received 13 July 2000 / Accepted 17 November 2000

\begin{abstract}
The small-amplitude Cepheid SU Cas and four members of the association Cas OB2 (HD 16893, HD 17327a and b, HD 17443) were investigated, using high-resolution CCD spectra. The following results were obtained: 1) All these objects have the same metallicity values, close to that of the Sun; 2) Elemental abundance indicates that SU Cas is a post first dredge-up star with an age from $110^{8}$ to $1.4510^{8} \mathrm{yr}$, and it is not crossing the Cepheid instability strip for the first time. The mean value of $\log g=2.35$ corresponds to pulsations in the fundamental tone, although errors in gravity estimations provide overtone pulsations. The questions about its pulsational mode and membership in Cas OB2 remained open; 3) HD 17327a is a slowly rotating HgMn-star with the highest helium content among such objects, while HD 16893 also has a manganese overabundance and might be classified as an Am-star; 4) HD 17327b and HD 17443 are rapidly rotating main-sequence stars, while HD 17443 has a helium content comparable with that of the Sun.
\end{abstract}

Key words. associations - stars: abundances - stars: Cepheids - stars: main-sequence

\section{Introduction}

The method of distance determination inside and outside our Galaxy by means of the period-luminosity-colour $(P-$ $L-C$ ) relation for Cepheids, is currently the most accurate one. Using the surface brightness method (Barnes et al. 1977), one can obtain Cepheid luminosities and distances through changes in their radii and colour-indices (which measure $\left.T_{\text {eff }}\right)$ during the pulsational periods. However, this process requires numerous, long-term spectroscopic observations. Moreover, additional difficulties arise from the brightness limit for spectroscopy: Cepheids with various values of pulsational period need to be studied, but the majority of them are comparatively faint objects.

A better method is to use Cepheids which belong to open clusters and associations. Although the majority of these are fainter than $8^{\mathrm{m}}$ (see Table 1), one can derive their luminosities through distances to these stellar groups.

Send offprint requests to: I. A. Usenko, e-mail: igus@deneb.odessa.ua
This method is based on matching the composite zeroage main sequence (ZAMS) and the colour-absolute magnitude diagram. The composite ZAMS is determined by the Hyades (or Pleiades) group parallax with addition of non-evolved main sequence (MS) stars from other, younger clusters, to their diagrams. Therefore, having multicolour (broad- or, better, narrow-band) photometric data for MS stars in an open cluster and using corresponding calibrations, one can derive distances and colourexcesses for these stars and use them to determine colourindices and luminosity of Cepheids from the same cluster.

At first, this is a very good, simple method. In reality, the discrepancy in determination of the distance modules for open clusters are, on average, $\pm 0 .{ }^{\mathrm{m}} 1-0{ }^{\mathrm{m}} 2$. This leads to a corresponding discrepancy in Cepheids luminosities of about $0{ }^{\mathrm{m}} 3-0 .{ }^{\mathrm{m}} 5$. It is obvious that all these $P-L-C$ relations have some important shortcomings, based on the following:

1) Inaccurate determination of colour-excesses, especially for Cepheids situated far from the open cluster field. 
2) The relationship of the distance calibration based on open clusters to the composite ZAMS. Such a ZAMS contains stars with a different degree of heavy element abundance, which has a very significant influence on the open cluster stars colour-indices and absolute magnitudes and on the ZAMS position in the diagram. For example, Hyades has a more metal rich content (1.5-2 times higher) than an average open cluster in the solar neighbourhood. The same can be said for the helium abundance.

3) The errors due to metallicity index determination from the multicolour (mainly broad-band) photometrical data. This problem is not limited only to Cepheids (the authors have often run across such cases, when $[\mathrm{Fe} / \mathrm{H}]$ values, determined by the model atmospheres method, have differed from the those determined using broad-band photometry, by $0.5-0.7 \mathrm{dex})$, - a similar situation also exists for the MS stars in open clusters.

4) The presence of a hot companion near a Cepheid. Such a presence might affect the Cepheid colour-index (especially $(B-V))$. According to Evans (1995) and Szabados (1992), the percentage of binary systems among classical Cepheids is from $21 \%$ to $50 \%$.

5) The Cepheid position on the Cepheid instability strip and the number of its crossings (Stift 1982).

In spite of these obstacles, open clusters and associations containing the Cepheids became a paradigm to investigations of the evolution of yellow supergiants, for comparisons of the observational data with theory predictions, and, moreover, for more precise definition of the $P-L-C$ relation calibration.

All the usefulness of the spectroscopic investigations of Cepheids and MS stars in open clusters lies in the fact that the MS B-stars are the Cepheids progenitors. Therefore, it would be possible to determine the helium abundance in open clusters and associations through its abundance in the atmospheres of MS B-stars and the lower level of the helium content in the atmospheres of Cepheids, which belong to these clusters and associations also the CNOelement abundances (as the key elements in the yellow supergiant evolution) in the Cepheids and MS stars atmospheres can be determined, and we can then perform their comparative analysis; the odd-elements ( $\mathrm{Na}$ and $\mathrm{Al}$ ) abundances can be verified, and as well as their connection to the luminosities of the Cepheids. The same can be performed for heavy $\alpha$-elements, iron group and $s$-process elements, and average metallicities for open clusters and associations can be obtained; $T_{\text {eff }}$ and $\log g$ values with a high precision are available and then the intrinsic colours and colour-excesses for Cepheids, and their masses can be specified. We can also check the influence of the different values of helium content and metallicity in calibrating Cepheids for $P-L-C$ relation.

Until recent time, such an investigation was not feasible. The main reason for that is the availability of only a small number of open clusters and associations that contain comparatively bright Cepheids and MS stars. This situation has since changed radically. Availability of large telescopes, equipped with echelle-spectrographs and CCD
Table 1. Galactic Cepheids in open clusters

\begin{tabular}{|c|c|c|c|c|}
\hline Cepheid & $\begin{array}{r}P \\
\text { (days) }\end{array}$ & $\begin{array}{l}V_{\text {mean }} \\
(\mathrm{mag})\end{array}$ & $\begin{array}{l}\text { Cluster or } \\
\text { Association }\end{array}$ & Comments \\
\hline SU Cas & 1.95 & 5.970 & Cas OB2 & DCEPS \\
\hline IR Cep & 2.11 & 7.784 & Per OB1 & DCEPS,(?) \\
\hline EV Sct & 3.09 & 10.137 & NGC 6664 & DCEPS \\
\hline SZ Tau & 3.15 & 6.531 & NGC 1647 & DCEPS,CM,NM \\
\hline BY Cas & 3.22 & 10.366 & NGC 663 & DCEPS, (?) \\
\hline GU Nor & 3.45 & 10.411 & NGC 6067 & DCEPS, CM,SC,(?) \\
\hline QZ Nor & 3.79 & 8.866 & NGC 6067 & DCEPS,CN,SC \\
\hline AH Vel & 4.22 & 5.695 & Cr 173 & $\mathrm{CM},(?)$ \\
\hline V1726 Cyg & 4.24 & 9.009 & Platais 1 & DCEPS \\
\hline CG Cas & 4.37 & 11.335 & NGC 7790 & $\mathrm{CM}, \mathrm{SC},(?)$ \\
\hline $\mathrm{CE}$ Cas b & 4.48 & 11.062 & NGC 7790 & \\
\hline CF Cas & 4.88 & 11.136 & NGC 7790 & \\
\hline CE Cas a & 5.14 & 10.922 & NGC 7790 & \\
\hline CV Mon & 5.38 & 10.299 & Anonym & \\
\hline V Cen & 5.49 & 6.836 & NGC 5662 & $\mathrm{CM}$ \\
\hline UY Per & 5.37 & 11.344 & King 4 & $\mathrm{CM}, \mathrm{SC}$ \\
\hline VY Per & 5.53 & 11.257 & $\mathrm{~h}, \chi$ Per & $\mathrm{SC}$ \\
\hline CS Vel & 5.90 & 11.687 & Rup 79 & \\
\hline V367 Sct & 6.29 & 11.596 & NGC 6449 & $\mathrm{DMC}, \mathrm{FP}$ \\
\hline BB Sgr & 6.64 & 6.947 & Coll 394 & $\mathrm{CM}$ \\
\hline U Sgr & 6.75 & 6.745 & M 25 & \\
\hline V440 Per & 7.57 & 6.282 & $h, \chi$ Per & CM,(?) \\
\hline DL Cas & 8.00 & 8.969 & NGC 129 & \\
\hline AC Mon & 8.01 & 10.067 & NGC 2323 & $(?)$ \\
\hline S Nor & 9.75 & 6.394 & NGC 6087 & \\
\hline AQ Car & 9.77 & 9.769 & Pup OB2 & $\mathrm{SC},(?)$ \\
\hline TW Nor & 10.79 & 11.704 & Lynga 6 & \\
\hline VX Per & 10.89 & 9.312 & $\mathrm{~h}, \chi$ Per & $\mathrm{SC}$ \\
\hline V340 Nor & 11.29 & 8.375 & NGC 6067 & \\
\hline SZ Cas & 13.63 & 9.853 & $\mathrm{~h}, \chi$ Per & DCEPS,SC \\
\hline VY Car & 18.99 & 7.443 & Car OB2 & $\mathrm{SC}$ \\
\hline RU Sct & 19.70 & 9.466 & Trump 35 & $\mathrm{CM}, \mathrm{SC}$ \\
\hline RZ Vel & 20.40 & 7.079 & Vel OB1 & $\mathrm{SC}$ \\
\hline WZ Sgr & 21.85 & 8.030 & C1814-191 & $\mathrm{SC}$ \\
\hline SW Vel & 23.44 & 8.120 & Vel OB5 & $\mathrm{SC}$ \\
\hline T Mon & 27.02 & 6.124 & Mon OB2 & $\mathrm{SC}, \mathrm{NM}$ \\
\hline KQ Sco & 28.69 & 9.807 & Sco OBa & $\mathrm{SC}$ \\
\hline U Car & 38.77 & 6.288 & Car OB2 & $\mathrm{SC}, \mathrm{NM}$ \\
\hline RS Pup & 41.39 & 6.947 & Pup OB3 & $\mathrm{SC},(?)$ \\
\hline SV Vul & 44.99 & 7.220 & Vul OB1 & $\mathrm{SC}, \mathrm{NM}$ \\
\hline GY Sge & 51.06 & 10.151 & OBanon & $\mathrm{SC}$ \\
\hline S Vul & 68.46 & 8.962 & Vul OB2 & $\mathrm{SC}$ \\
\hline
\end{tabular}

DCEPS - s-Cepheid.

$\mathrm{CM}$ - coronae member.

$\mathrm{SC}$ - stellar complex.

FP - fundamental period.

NM - non-member according to Gieren, Fouque \& Gomez (1997).

(?) - membership in cluster (association) needs confirmation.

detectors allow one to obtain high-resolution spectra with a high $S / N$ ratio for objects as faint as $14^{\mathrm{m}}$. Since these developments, the tasks mentioned above have become more feasible.

With this paper, we start a series of publications devoted to investigations of Cepheids and MS stars in selected Galactic open clusters.

\section{Association Cas OB2}

The association Cas OB2, in spite of its small population, is significant, because it contains the 1.95 bright smallamplitude Cepheid (DCEPS) SU Cas, which is a calibrating object for the $P-L-C$ - relation with the lowest value of the pulsational period.

The investigation of this association has a comparatively short history. First, Racine (1968), who suggested the existence of an $\mathrm{OB}-$ association on the basis of the association of some B-stars (HD 17327, HD 17443, HD 17706) with the dust complex, noted that $\mathrm{SU}$ Cas is not a member 
of the association, though it does illuminate a reflection nebulosity (a segment of a dusty path along Orion's arm) in this complex.

The real discussion about the luminosity and corresponding distance value for SU Cas has arisen due to the differences in its mean radius, determined by various methods and authors. The use of the traditional Baade-Wesselink (BW) method produced values near 19.1-21.8 $R_{\odot}$, whereas the maximum likelihood (ML) method and pulsational analysis (PA) resulted in values from $29 R_{\odot}$ to $40.3 R_{\odot}$. The minimum radius of $18.6 R_{\odot}$ was determined by means of the surface brightness (SB) method by Niva \& Schmidt (1979) (see Table 1 from Gieren 1982). It is clear that such a disagreement in the radius might be interpreted as pulsational in fundamental tone or even in first and second overtone.

The problems mentioned above were resolved by Turner \& Evans (1984). They investigated in detail all stars below $14^{\mathrm{m}}$ in the vicinity of SU Cas with membership in the Cas OB2 association. They found five stars to be association members: HD 16893, HD 17327a, HD 17327b, HD 17443 and HD 23475. A distance of $258 \pm 3 \mathrm{pc}$ found for SU Cas with the colour-excess $E_{B-V}=0.27$ and $T_{\text {eff }}=$ $6383 \mathrm{~K}$ gave an absolute magnitude of about -1 . 94 . This value is in good agreement with the $P-L$ relation for galactic Cepheids pulsating in the fundamental tone. The mean radius of SU Cas derived from these data is $18.2 \pm$ $0.9 R_{\odot}$ (with an account of a close companion A0-A5 V presence based on $I U E$ observations).

As is seen, such a radius value, derived by Turner \& Evans (1984), is in good agreement with that determined by the SB method (Niva \& Schmidt 1979) and is close to the $\mathrm{BW}$ radius values. It also is significantly difference to the ML and PA data. This might be explained partly by the presence of a hot companion, mentioned above. Such a companion (A0-A5 V, Turner \& Evans 1984; A0 V, Usenko 1990; B9.5 V, Evans 1991), with an orbital period of about 462. 5 (Szabados 1991) and brightness differences $\Delta V=0.109$ and $\Delta(B-V)=0.072$ (Gorynya et al. $1996)$, affects the colour-indices of SU Cas, especially ( $B-$ $V)_{0}$, and the radial velocity curve during orbital motion. Since the SB method used $(V-R)_{0}$ colour-indices, for which influence of the hot companion is insignificant, the Niva \& Schmidt (1979) radius value is the closest to that of Turner \& Evans (1984).

Nevertheless, Evans (1991), using the $I U E$ spectra of the SU Cas blue companion (B9.5 V) arrived at an unexpected value of the Cepheid's absolute magnitude, -3 . $^{\mathrm{m}} 14$. This result was confirmed by recent measurements of the SU Cas trigonometric parallax (2.31 \pm 0.54 milliarcsec $)$ using the HIPPARCOS satellite data. It gives a distance of $433_{-82}^{+132}$ pc (Szabados 1997) and $M_{V}=-3.25 \pm 0.98$ for $E_{B-V}=0.287$ (Fernie et al. 1995). Therefore, SU Cas is placed by a factor of 1.7 farther than the association stars and is not a member of Cas OB2.

Using the mean value of $T_{\text {eff }}=6300 \mathrm{~K}$, taken from Luck \& Lambert (1981) and Andrievsky et al. (1996) (determined from spectroscopic analysis using the model at- mospheres method), we can obtain a radius value equal to $33.8 R_{\odot}$. This value corresponds to pulsation in the second overtone (Gieren 1982). Thus, the same problem calls for a new solution.

Nevertheless, the HIPPARCOS distance for SU Cas is close to that the nearby the same for background association with HD 17706 (401 \pm 38 pc from Turner \& Evans 1984), which is not a member of Cas OB2. Besides, using polarimetric observations, Pavlova \& Rspaev (1986) have detected unusual changes in the polarization degree with wavelength for HD 17443 and SU Cas. They explained this effect by the presence of S-shaped fibres in the nebulosity structure which have the same orientation as the polarization vector. The polarization positional angles for SU Cas and HD 17443 are close to each other. The polarization degree for SU Cas in the $V$-band is somewhat larger than for other stars, probably due to mass loss through the stellar wind (Welch \& Duric 1988). All field stars in this region show polarization vectors almost parallel to the galactic plane.

\section{Selection of the stars and observational material}

In our observational program we have included SU Cas (F6 IIb-F8 IIb) itself and four stars, members of Cas OB2 from the Turner \& Evans (1984) list: HD 16893 (A3 Vp), HD 17327a and b (B8 III and A2 Vn) and HD 17443 (B9 $\mathrm{V})$. Except for SU Cas, each star from this list is interesting itself: HD 16893, according to Turner \& Evans (1984), is a spectroscopic binary; HD 17327a and b are components of a visual binary ADS 2142, in which HD 17327a is located near the turn-off point of Cas OB2 (see Fig. 5 in Turner \& Evans 1984); HD 17443 is clearly embedded in the same dust cloud as SU Cas.

The high-resolution spectra of these stars were obtained with the echelle-spectrometers PFES (Panchuk et al. 1998) and LYNX (Panchuk et al. 1999) at the 6-m telescope of the Special Astrophysical Observatory, Russian Academy of Sciences (Russia, Northern Caucasus). The resolving power was 14000 and 25000 , respectively, $S / N \approx 70-100$. The information concerning the program stars and their CCD spectra is given in Table 2.

Using the MIDAS software, we extracted spectra from CCD frames, made dark and cosmic ray hit subtraction, and wavelength calibration. The line equivalent widths $\left(W_{\lambda}\right)$ were determined using the DECH20 code (Galazutdinov 1992). In our analysis we did not use lines with $W_{\lambda} \gtrsim 150 \mathrm{~m} \AA$. The accuracy of the equivalent widths is of the order of $5-10 \%$. This estimate is based on the comparison of the values derived from lines present in the overlapping spectral orders.

Before the atmospheric parameter determination and abundance calculations we made visual inspections of the program stars spectra (excluding SU Cas), because some of them have high projected rotational velocities. As an example we demonstrate in Fig. 1 two fragments of the 
Table 2. Program stars and their CCD spectra

\begin{tabular}{lccccc}
\hline Star & $\begin{array}{c}\text { Spectrum } \\
\text { No. }\end{array}$ & $\begin{array}{c}\text { HJD } \\
2450000+\end{array}$ & $\begin{array}{c}V \\
(\mathrm{mag})\end{array}$ & $\begin{array}{c}\text { Region } \\
(\AA)\end{array}$ & $\begin{array}{c}\text { Exposure } \\
(\mathrm{min})\end{array}$ \\
\hline SU Cas(1) & $\mathrm{s} 20716$ & 1003.5000 & $5.70-$ & $4682-8596$ & 10 \\
SU Cas(2) & $\mathrm{s} 21012$ & 1006.4960 & -6.18 & $4420-7764$ & 10 \\
HD 16893 & $\mathrm{s} 21011$ & 1006.5280 & 8.53 & $4682-8596$ & 20 \\
HD 17327a & $\mathrm{s} 20715$ & 1003.5270 & 7.53 & $4420-7767$ & 20 \\
& $\mathrm{~s} 23204$ & 1246.1720 & & $4383-7960$ & 13 \\
& $\mathrm{~s} 23205$ & 1246.1790 & & $4383-7960$ & 17 \\
HD 17327b & $\mathrm{s} 23206$ & 1246.2020 & 10.33 & $4383-7960$ & 30 \\
HD 17443 & $\mathrm{s} 20714$ & 1003.4800 & 8.74 & $4420-7767$ & 30 \\
\hline
\end{tabular}
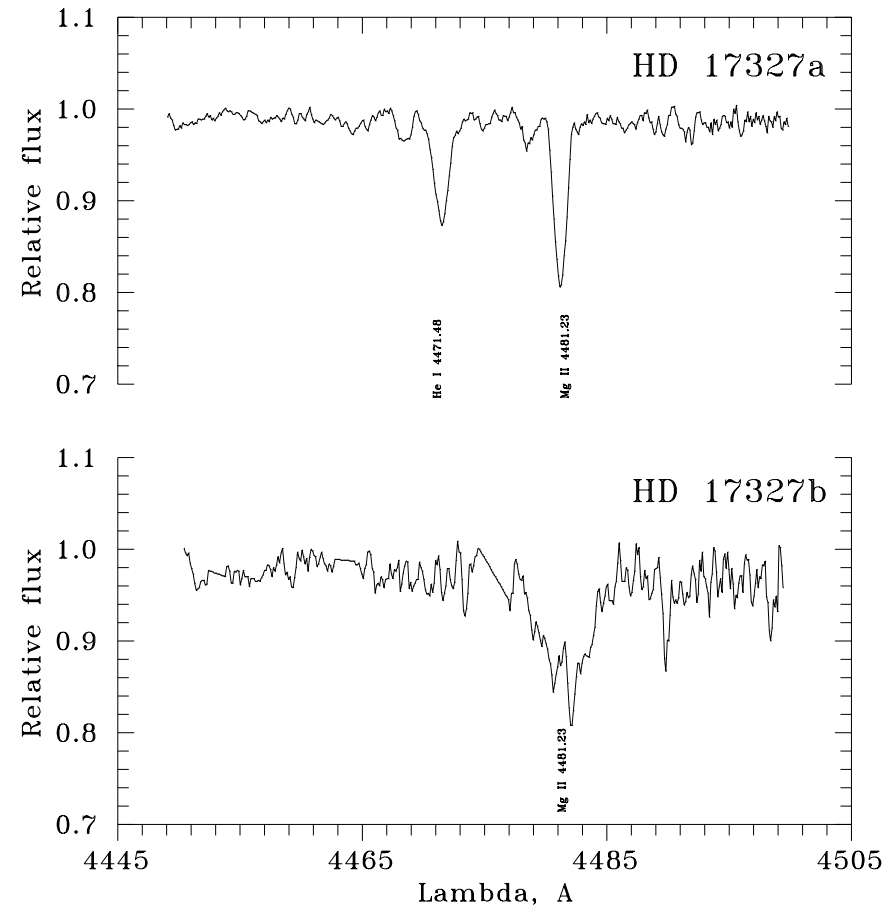

Fig. 1. Fragments of spectra for HD 17327a and b in the vicinity of the He I $4471 \AA$ and Mg II $4481 \AA$ lines

same spectral region (He I $4471 \AA$ and Mg II $4481 \AA$ lines) for two components of the visual binary ADS 2142, HD 17327a and b.

To evaluate line blends for objects with high projected rotational velocities, the spectral synthesis technique was applied. This was performed with the help of the SYNSPEC code (Hubeny et al. 1994). For SU Cas we used $v \sin i$ from Takeda et al. (1997). Projected rotational velocities for program stars were estimated by fitting the synthetic spectrum to the observed one. The results are given in Table 3.

Figure 2 shows the observed and synthetic spectra for the $\mathrm{H} \alpha$ region of $\mathrm{HD} 17443$. As is seen, $\mathrm{HD} 17443$ is an ordinary main-sequence B-star.
Table 3. Projected rotational velocities for program stars

\begin{tabular}{lcc}
\hline Star & $\begin{array}{c}v \sin i \\
\left(\mathrm{~km} \mathrm{~s}^{-1}\right)\end{array}$ & Remarks \\
\hline SU Cas & 10 & Spectroscopic binary \\
HD16893 & 25 & Primary component of ADS2142 \\
HD 17327a & 40 & Secondary component of ADS 2142 \\
HD 17327b & 200 & in the same dust cloud as SU Cas \\
HD 17443 & 180 & \\
\hline
\end{tabular}

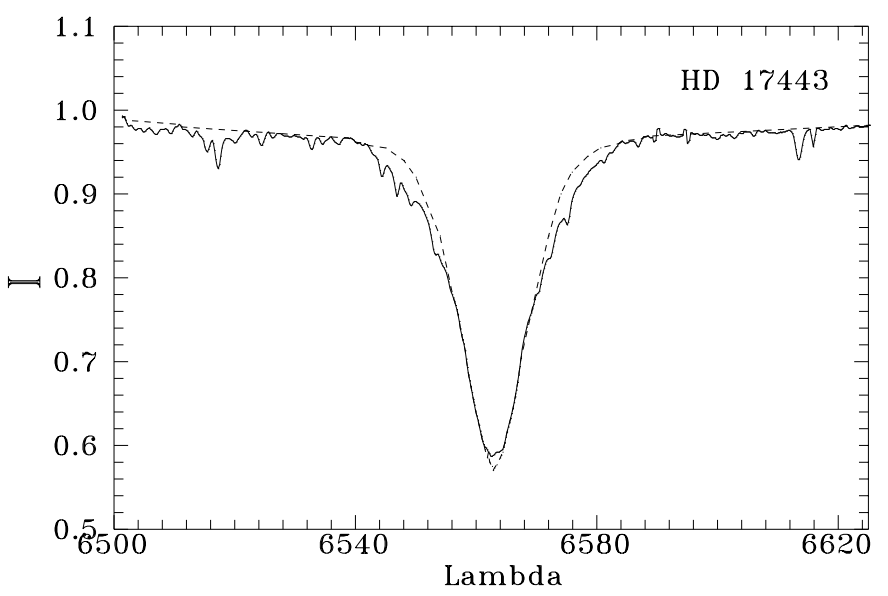

Fig. 2. The synthetic and observed profiles of $\mathrm{H}_{\alpha}$ in the spectrum of HD 17443

\section{Atmospheric parameters and chemical composition}

\subsection{Atmospheric parameters}

Determination of the atmospheric parameters of the Cas OB2 objects was performed by different methods. For SU Cas, as a variable yellow supergiant, we obtained values of $T_{\text {eff }}$ based on the ratio of the spectral line depths (Kovtyukh et al. 1998). This method, based on spectroscopic criteria only, allows us to estimate these values with an accuracy of 50-80 K.

For other program stars we used:

1) $(U-B),(B-V)-\left(T_{\text {eff }}, \log g\right)$ calibrations (Castelli 1991);

2) $(b-y), m 1, c 1, \beta-\left(T_{\text {eff }}, \log g\right)$ calibrations (Moon \& Dworetsky 1985; Napivotzki et al. 1993);

3) $(b-y)-\left(T_{\text {eff }}, \log g\right)$ calibrations (Castelli 1991); 
Table 4. $T_{\text {eff }}$ and $\log g$ determination

\begin{tabular}{|c|c|c|c|c|c|c|c|c|c|c|c|}
\hline Star & Phase & $\begin{array}{c}T_{\text {eff }} \\
\text { (ratio) }\end{array}$ & $\begin{array}{c}T_{\text {eff }} \\
(B-V)_{0}\end{array}$ & $\begin{array}{c}T_{\text {eff }} \\
(U-B)_{0}\end{array}$ & $\begin{array}{c}T_{\text {eff }} \\
(u b v y \beta)\end{array}$ & $\begin{array}{c}T_{\text {eff }} \\
\left(\mathrm{H}_{\alpha}\right)\end{array}$ & $\begin{array}{c}T_{\text {eff }} \\
\left(\mathrm{H}_{\beta}\right)\end{array}$ & $\begin{array}{c}\log g \\
(u b v y \beta)\end{array}$ & $\begin{array}{l}\log g \\
\left(\mathrm{H}_{\alpha}\right)\end{array}$ & $\begin{array}{l}\log g \\
\left(\mathrm{H}_{\beta}\right)\end{array}$ & $\begin{array}{l}\log g \\
(\mathrm{Fe})\end{array}$ \\
\hline SU Cas (1) & 0.498 & 6200 & - & - & - & - & - & - & - & - & 2.30 \\
\hline SU Cas (2) & 0.965 & 6450 & - & - & - & - & - & - & - & - & 2.40 \\
\hline HD 16893 & - & - & 8500 & - & - & - & - & - & - & - & 4.00 \\
\hline HD $17327 a$ & - & - & 12400 & 12500 & 12450 & 12000 & 12000 & 3.40 & 3.20 & 3.20 & - \\
\hline HD $17327 \mathrm{~b}$ & - & - & 7800 & 7800 & - & 10000 & 10000 & - & 4.00 & 4.00 & - \\
\hline HD 17443 & - & - & 11000 & 11000 & 10800 & 10900 & 10900 & 4.05 & 4.05 & 4.05 & - \\
\hline
\end{tabular}

Phases were calculated according to Berdnikov \& Pastukhova (1994).

Table 5. Adopted atmospheric parameters

\begin{tabular}{lcccc}
\hline Star & Phase & $T_{\text {eff }}$ & $\log g$ & $V_{t}$ \\
\hline SU Cas (1) & 0.498 & 6200 & 2.30 & 3.3 \\
SU Cas (2) & 0.965 & 6450 & 2.40 & 3.3 \\
HD 16893 & - & 8500 & 4.00 & 3.0 \\
HD 17327a & - & 11700 & 3.20 & 1.0 \\
HD 17327b & - & 10000 & 4.00 & 3.0 \\
HD 17443 & - & 10900 & 4.05 & 3.0 \\
\hline
\end{tabular}

4) Comparisons of the observed $\mathrm{H}_{\alpha}$ and $\mathrm{H}_{\beta}$ line profiles with the synthetic spectra.

The $(B-V)$ and $E_{B-V}$ data were taken from Turner \& Evans (1984), the $(b-y)$ and $E_{b-y}$ data from Schmidt (1978). All the preliminary $T_{\text {eff }}$ determinations (with a mean uncertainty of $100 \mathrm{~K}$ ) are given in Table 4 .

For cooler stars, SU Cas and HD 16893, the surface gravities were determined assuming equal abundances of Fe I and Fe II. The preliminary $\log g$ determinations (with mean uncertainties of \pm 0.15 and \pm 0.2 dex, respectively) are given in Table 4.

The microturbulent velocities $\left(V_{\mathrm{t}}\right)$ for $\mathrm{SU}$ Cas, HD 16893 and HD 17327a were obtained assuming the abundances from Fe II lines to be independent of the equivalent line widths with a mean uncertainty of $0.25 \mathrm{~km} \mathrm{~s}^{-1}$. For HD 17443 the corresponding value of $V_{\mathrm{t}}=3 \mathrm{~km} \mathrm{~s}^{-1}$ was adopted as a more appropriate one for late B stars. Finally, adopted atmospheric parameters are listed in Table 5.

\subsection{Analysis technique}

The analysis was carried out using our implementation of Kurucz's WIDTH9 code. Atmosphere models were interpolated from the Kurucz (1992) grid. All the oscillator strengths were taken from various sources. For SU Cas we used so-called "solar" $\log g f$ values (Kovtyukh $\&$ Andrievsky 1999). They were derived by us using unblended solar lines (solar spectrum by Kurucz et al. 1984).

For B- and A-type stars we used oscillator strengths from the Kurucz (1995) database (CD-ROM 15, 18). For HD 16893 we also used additional data from Adelman et al. (1996)

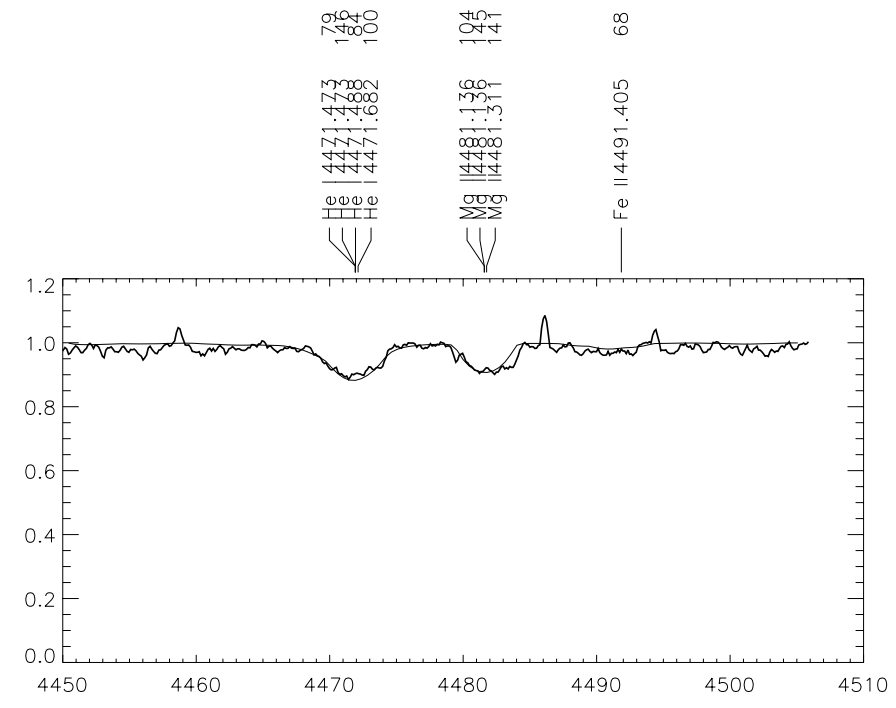

Fig. 3. The observed and synthetic spectra for the HD 17443 region near the He I $4471 \AA$ and $\mathrm{Mg}$ II $4481 \AA$ lines

\subsection{Abundances for the Cas OB2 members}

In Tables 6, 7 and 8 we give the calculated abundances for SU Cas, HD 17327a and HD 16893, respectively. It should be noted that for the rapidly rotating B-star HD 17443 we estimated only helium and magnesium abundance from two strong lines He I $4471 \AA$ and Mg II $4481 \AA$. This was performed using spectral synthesis (see Fig. 3). In this case, helium and magnesium abundances are solar.

From the data shown in Table 6 one can conclude that SU Cas has a carbon deficit, a nitrogen overabundance, and a solar-like oxygen content. Sodium and aluminium are in a slight overabundance, while the $\alpha$ - and Fe-group elements content is close to solar. Some heavy $s$ process elements demonstrate a small overabundance (see Fig. 4). All these data are in good agreement with those of Andrievsky et al. (1996). Therefore, SU Cas is not crossing the Cepheids instability strip for the first time.

According to Tables 5 and 7 , one can notice that HD 17327a has the atmospheric parameters and chemical composition typical of mercury - manganese stars: some overabundance of carbon, solar-like or deficient nitrogen and oxygen content, a very noticeable deficit of $\mathrm{Mg}$ and $\mathrm{Si}$, and an overabundance of P, S, Mn, Y, Zr, and $\mathrm{Hg}$ (see Fig. 5). The overwhelming majority of these chemically 
Table 6. Abundances for SU Cas

\begin{tabular}{|c|c|c|c|c|}
\hline \multirow[t]{2}{*}{ Element } & \multicolumn{3}{|c|}{ Average } & \multirow{2}{*}{$\begin{array}{c}\text { Paper II } \\
\text { (Average) }\end{array}$} \\
\hline & {$[\mathrm{El} / \mathrm{H}]$} & $\sigma$ & NL & \\
\hline CI & -0.27 & 0.17 & $8-11$ & - \\
\hline $\mathrm{N} \mathrm{I}$ & +0.53 & 0.09 & $3-3$ & - \\
\hline O I & -0.01 & 0.04 & $0-2$ & - \\
\hline $\mathrm{Na} \mathrm{I}$ & +0.25 & 0.07 & $2-4$ & +0.50 \\
\hline $\mathrm{Mg} \mathrm{I}$ & -0.18 & 0.22 & $1-4$ & -0.05 \\
\hline $\mathrm{Al} \mathrm{I}$ & +0.20 & 0.08 & $3-3$ & - \\
\hline Si I & +0.09 & 0.11 & $16-31$ & - \\
\hline Si II & -0.01 & - & $1-1$ & - \\
\hline S I & +0.19 & 0.16 & $4-5$ & -0.30 \\
\hline $\mathrm{Ca} \mathrm{I}$ & -0.02 & 0.19 & $9-10$ & +0.04 \\
\hline Sc II & +0.05 & 0.17 & $6-9$ & -0.05 \\
\hline Ti I & +0.11 & 0.22 & $12-20$ & +0.11 \\
\hline Ti II & -0.00 & 0.15 & $2-8$ & -0.01 \\
\hline V I & +0.10 & 0.09 & $3-4$ & +0.10 \\
\hline V II & -0.04 & 0.08 & $2-5$ & -0.05 \\
\hline Cr I & -0.05 & 0.23 & $4-17$ & -0.05 \\
\hline Cr II & -0.04 & 0.14 & $8-12$ & -0.03 \\
\hline Mn I & -0.34 & 0.19 & $7-8$ & -0.17 \\
\hline $\mathrm{FeI}$ & -0.03 & 0.13 & $152-182$ & -0.10 \\
\hline Fe II & -0.03 & 0.10 & $18-28$ & -0.10 \\
\hline Co I & -0.03 & 0.20 & $4-6$ & +0.10 \\
\hline Ni I & -0.06 & 0.21 & $33-63$ & -0.10 \\
\hline $\mathrm{Cu} \mathrm{I}$ & -0.26 & 0.47 & $1-3$ & - \\
\hline Zn I & -0.23 & 0.30 & $1-2$ & -0.09 \\
\hline Y II & +0.01 & 0.17 & $6-7$ & - \\
\hline Zr II & +0.06 & 0.21 & $2-5$ & +0.35 \\
\hline Ba II & +0.17 & - & $0-1$ & - \\
\hline La II & +0.30 & 0.08 & $1-2$ & +0.08 \\
\hline Ce II & -0.04 & - & $3-6$ & +0.32 \\
\hline PrII & -0.66 & 0.09 & $1-2$ & +0.01 \\
\hline Nd II & +0.06 & 0.21 & $3-8$ & +0.22 \\
\hline $\mathrm{Eu} \mathrm{II}$ & +0.24 & 0.05 & $1-2$ & - \\
\hline Gd II & +0.28 & - & $0-1$ & +0.26 \\
\hline
\end{tabular}

Paper II - data from Andrievsky et al. (1996).

NL - minimal and maximal number of lines.

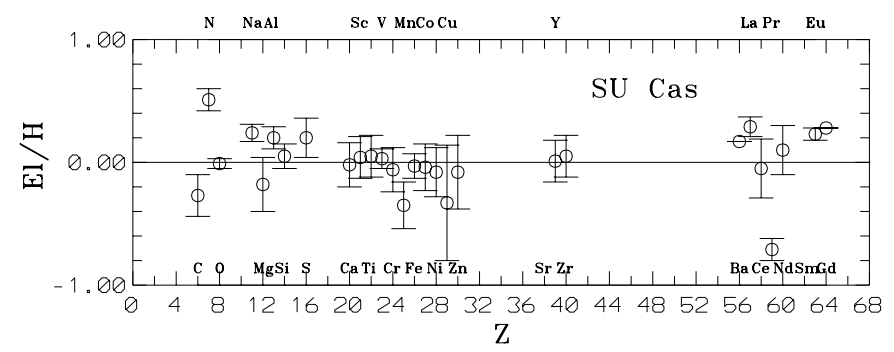

Fig. 4. Elemental abundance for SU Cas

peculiar stars belong to binary systems. In 39 such systems they are primary components (Batten et al. 1989; Lebedev 1987).

As is known, single HgMn-stars or primary components of SB1 binaries belong to the classical group $(\log (\mathrm{Mn} / \mathrm{Fe}) \approx 0$ dex $)$, while SB2 ones belong to the Searl-Sargent group $(\log (\mathrm{Mn} / \mathrm{Fe}) \approx-1.0 \mathrm{dex})$ (Searle \& Sargent 1967; Ryabchikova et al. 1996). In Fig. 6 we show the positions of HD 17327a on the $\log (\mathrm{Mn} / \mathrm{H})-T_{\text {eff }}$
Table 7. Average abundance for HD 17327a

\begin{tabular}{lccc}
\hline Element & {$[\mathrm{El} / \mathrm{H}]$} & $\sigma$ & $\mathrm{NL}$ \\
\hline He I & +0.24 & - & $1-2$ \\
C I & +0.56 & 0.31 & $1-3$ \\
N I & +0.31 & - & $0-1$ \\
O I & -0.12 & 0.35 & $2-5$ \\
Mg I & -0.51 & - & $1-1$ \\
Mg II & -0.21 & 0.44 & $1-3$ \\
Al II & -0.36 & - & $0-1$ \\
Si II & -0.60 & 0.26 & $6-6$ \\
P II & +1.02 & - & $1-1$ \\
S II & +0.34 & 0.18 & $1-3$ \\
Sc II & +1.30 & - & $1-1$ \\
Ti II & +0.58 & 0.36 & $4-9$ \\
Cr II & +0.46 & 0.31 & $9-15$ \\
Mn II & +1.89 & 0.23 & $11-18$ \\
Fe II & -0.10 & 0.24 & $26-66$ \\
Y II & +1.87 & - & $1-2$ \\
Hg I & +4.39 & - & $1-1$ \\
\hline
\end{tabular}

$\mathrm{NL}$ - minimal and maximal number of lines.

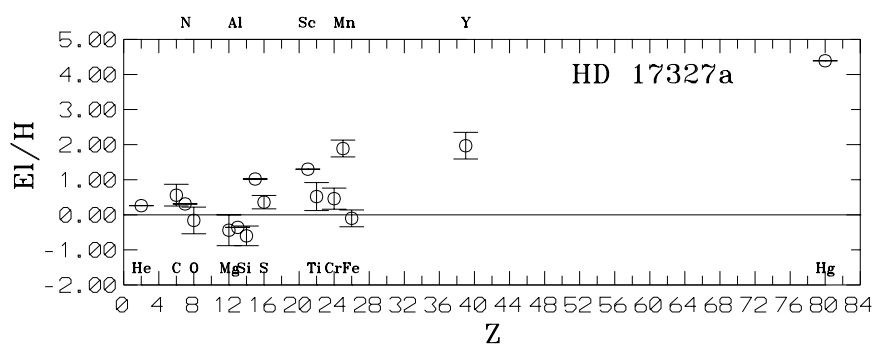

Fig. 5. Elemental abundance for HD 17327a

relation (Ryabchikova 1997). A good agreement with this relation is noticeable.

In Fig. 7 the position of HD 17327a on the graph is located within the classical HgMn-star group.

The most interesting feature of this star is the helium overabundance, while the main-sequence B star HD 17443 has a solar content. This fact can be due to the position of HD 17327a near the turn-off point for Cas OB2. Helium overabundance for this star is not a result of atmosphere enrichment by the products of the CNO-cycle (in this case the $\mathrm{N} / \mathrm{C}$ ratio would reach $1.0 \mathrm{dex}$ and more, while for HD 17327 a this ratio is $\approx 0.7 \mathrm{dex}$ ), but it is more likely as a result of the so-called light-induced drift (LID) mechanism (Atutov \& Shalagin 1988).

On the other hand, the results for HD 16893 are very interesting, too (Table 8). We can say that carbon has a noticeable deficit, while nitrogen and oxygen have a solarlike content. This is very unusual. It is seen that the abundances of $\mathrm{Al}, \mathrm{Mg}$ and $\mathrm{Si}$ in $\mathrm{HD} 16893$ are opposite to those of HD 17327a. The overabundance of Mn is of a factor of 3 less and the iron abundance is the same as that for HD 17327a. But content of the $s$-process elements is rather high (see Fig. 8). Moreover, $\log (\mathrm{Mn} / \mathrm{H})$ and $\log$ $(\mathrm{Mn} / \mathrm{Fe})$ are comparable to those of SB2 stars (see Fig. 6 


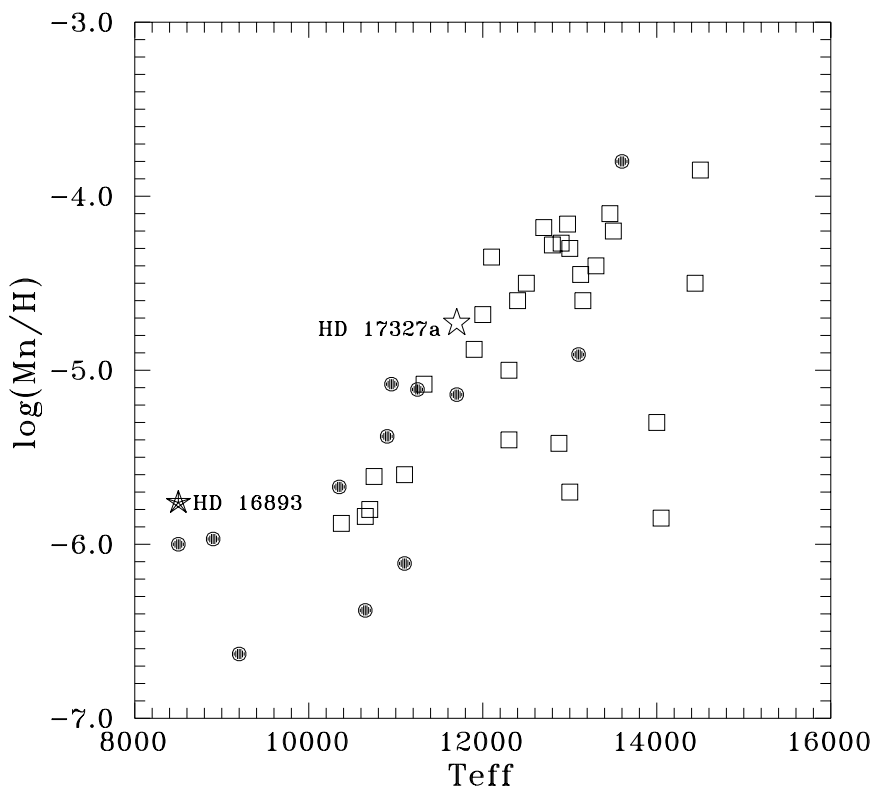

Fig. 6. The manganese abundance relation from $T_{\text {eff }}$ in atmospheres of HgMn stars. Open squares represent classical and SB1 HgMn stars, filled circles - Searle-Sargent (SB2) group HgMn stars, the open star indicate HD 17327a, while the filled star, - HD 16893

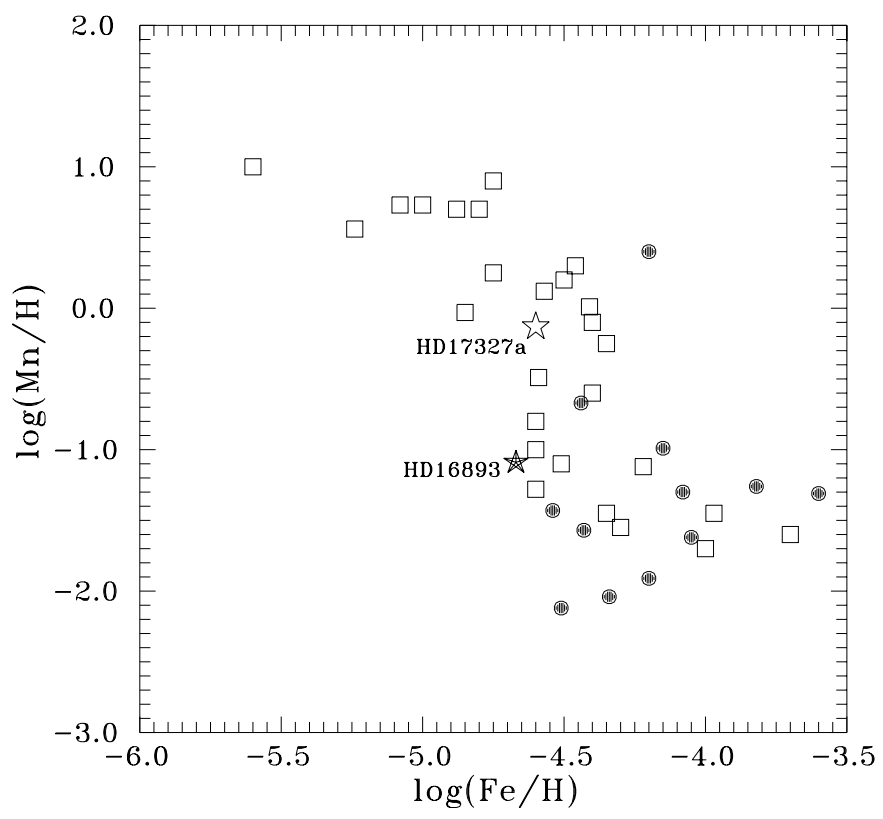

Fig. 7. The $\log g(\mathrm{Mn} / \mathrm{Fe})$ vs. $\log (\mathrm{Fe} / \mathrm{H})$ abundances relation. All symbols are the same as in Fig. 6

and Fig. 7). It would be reasonable to check this object for a presence of a strong magnetic field.

\section{Luminosities, radii and masses}

To estimate the luminosities and radii of the Cas OB2 objects we can use $T_{\text {eff }}$ determined spectroscopically and $M_{V}$ taken from Turner \& Evans (1984). Since we have obtained two spectrograms for SU Cas near the brightness minimum and maximum, the mean $T_{\text {eff }}=6325 \mathrm{~K}$ was used.
Table 8. Abundances for HD 16893

\begin{tabular}{|c|c|c|c|c|c|c|}
\hline \multirow[t]{2}{*}{ Element } & \multicolumn{3}{|c|}{$\log g f$ (Kurucz) } & \multicolumn{3}{|c|}{$\log g f$ (Adelman et al.) } \\
\hline & {$[\mathrm{El} / \mathrm{H}]$} & $\sigma$ & NL & {$[\mathrm{El} / \mathrm{H}]$} & $\sigma$ & NL \\
\hline $\bar{C}$ I & -0.45 & 0.17 & 7 & -0.50 & 0.18 & 5 \\
\hline N I & +0.09 & 0.03 & 2 & -0.20 & 0.51 & 5 \\
\hline O I & -0.12 & - & 1 & -0.07 & 0.18 & 4 \\
\hline $\mathrm{Na} I$ & +0.29 & - & 1 & - & - & - \\
\hline Mg I & -0.98 & 0.22 & 2 & -0.58 & - & 1 \\
\hline Mg II & - & - & - & +0.54 & - & 1 \\
\hline Al I & +0.92 & 0.08 & 2 & +0.92 & 0.08 & 2 \\
\hline Si II & -0.45 & - & 1 & -0.14 & 0.38 & 3 \\
\hline S I & +0.20 & 0.20 & 3 & +0.20 & 0.20 & 3 \\
\hline $\mathrm{Ca} \mathrm{I}$ & -0.02 & 0.23 & 15 & - & - & - \\
\hline Sc II & +0.04 & 0.38 & 6 & -0.12 & 0.12 & 2 \\
\hline Ti I & - & - & - & +0.37 & 0.26 & 2 \\
\hline Ti II & -0.20 & 0.15 & 13 & -0.05 & 0.28 & 13 \\
\hline V II & +0.78 & - & 1 & +0.78 & - & 1 \\
\hline Cr II & -0.09 & 0.15 & 10 & -0.03 & 0.24 & 10 \\
\hline Mn I & +0.65 & 0.24 & 4 & - & - & - \\
\hline Mn II & - & - & - & +1.05 & 0.02 & 2 \\
\hline $\mathrm{Fe} \mathrm{I}$ & -0.21 & 0.17 & 18 & -0.68 & - & 1 \\
\hline Fe II & -0.17 & 0.09 & 10 & -0.14 & 0.42 & 13 \\
\hline Co I & +1.10 & 0.18 & 5 & +1.19 & 0.26 & 6 \\
\hline Ni I & +0.06 & 0.20 & 8 & - & - & - \\
\hline $\mathrm{Cu} \mathrm{I}$ & +0.22 & - & 1 & +0.22 & - & 1 \\
\hline Zn I & -0.01 & 0.27 & 2 & - & - & - \\
\hline Sr I & +1.43 & - & 1 & - & - & - \\
\hline Y I & +2.56 & - & 1 & - & - & - \\
\hline Y II & +0.58 & 0.49 & 5 & +0.41 & 0.34 & 3 \\
\hline Zr II & +0.63 & 0.28 & 3 & +0.63 & 0.28 & 3 \\
\hline Ba II & +0.23 & 0.42 & 4 & +0.35 & - & 1 \\
\hline La II & +1.14 & 0.01 & 2 & +1.14 & 0.01 & 2 \\
\hline Ce II & +1.21 & 0.43 & 3 & +1.43 & 0.56 & 4 \\
\hline Pr II & - & - & - & +1.72 & - & 1 \\
\hline Nd II & +1.85 & - & 1 & +1.85 & - & 1 \\
\hline Sm II & +1.90 & - & 1 & - & - & - \\
\hline
\end{tabular}

NL - number of lines.

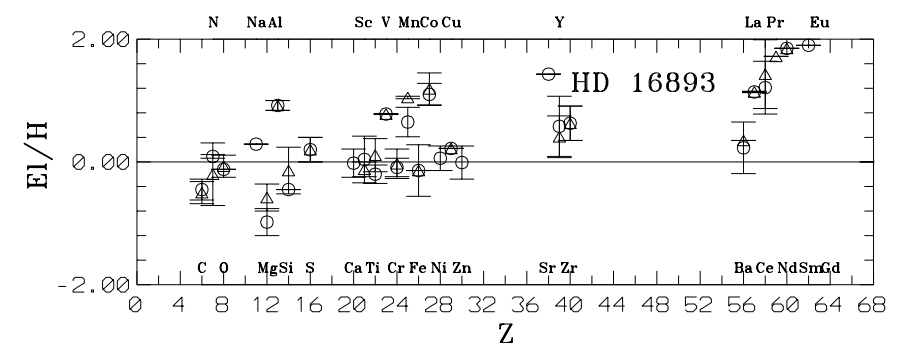

Fig. 8. Elemental abundance for HD 16893. Circles, - data, obtained using Kurucz (1995) log $g f$ values, triangles, - using Adelman et al. (1996) ones

We have also used bolometric corrections from Strajzys (1982) for all the stars, excluding SU Cas. Moreover, in case of SU Cas we have also used its absolute magnitude from Szabados (1997).

Evolutionary masses for Cas OB2 members were determined by various methods. According to the CNOabundance data, SU Cas is an object in the post red 


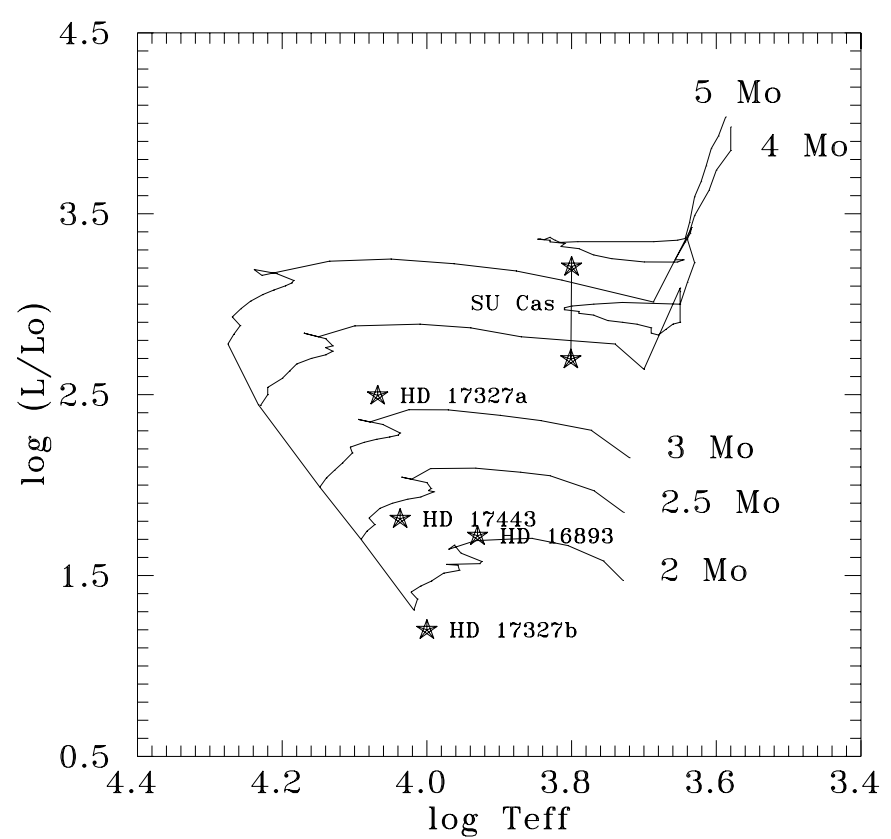

Fig. 9. HR diagram for Cas OB2 members. Evolutionary tracks from Schaller et al. (1992) recalculated for $Z=0.02$

Table 9. Luminosities, radii and masses for the Cas OB2 members

\begin{tabular}{lccccc}
\hline Star & $M_{\text {bol }}$ & $\log \left(L / L_{\odot}\right)$ & $R / R_{\odot}$ & $M_{\text {ev }}$ & $M_{\text {in }}$ \\
\hline SU Cas (1) & -1.94 & 2.696 & 18.6 & 3.7 & - \\
SU Cas (2) & -3.25 & 3.208 & 34.5 & 5.2 & - \\
HD 16893 & +0.44 & 1.720 & 3.4 & 2.1 & - \\
HD 17327a & -1.50 & 2.496 & 4.3 & 3.3 & - \\
HD 17327b & +1.74 & 1.200 & 1.3 & - & 2.0 \\
HD 17443 & +0.21 & 1.814 & 2.3 & - & 2.8 \\
\hline
\end{tabular}

(1) $-M_{V}$ from Turner \& Evans (1984).

(2) - from Szabados (1997).

supergiant evolutionary phase, and we can use the equation from Chiosi et al. (1992) for a case of mild overshooting:

$\log \left(L / L_{\odot}\right)=3.52 \log \left(M / M_{\odot}\right)+0.7$

For other objects the evolutionary and initial mass estimations were obtained from their positions in the HR diagram with the evolutionary tracks from Schaller et al. (1992) (recalculated using an interpolation for the metallicity value $Z=0.02$ ) for stars with $2 M_{\odot}, 2.5 M_{\odot}$, $3 M_{\odot}, 4 M_{\odot}$, and $5 M_{\odot}$, respectively. All these evolutionary tracks in the HR diagram and the positions of the Cas OB2 members are given in Fig. 9. Their bolometric magnitudes $M_{\text {bol }}$, luminosities $L$, radii $R$ and evolutionary $M_{\mathrm{ev}}$ and initial $M_{\text {in }}$ masses are given in Table 9 . For the two MS-stars we can apply the following relation:

$\log \left(L / L_{\odot}\right) \approx 4 \log \left(M / M_{\odot}\right)$

\section{Identification of the pulsation mode for SU Cas and its membership in the association Cas OB2}

According to the conclusions derived in 2, the main question arising is whether SU Cas is a member of Cas OB2 with a pulsation in the fundamental tone (Turner \& Evans 1984), or it is a Cepheid pulsating in an overtone (Evans 1991; Szabados 1997)? The first conclusion was based on photometric and radial velocity data, while the second one on the distance value determined from the hot companion parameters and on the HIPPARCOS parallax. It should be noted that Evans obtained $M_{V} \sim-3^{\mathrm{m}} 14$ from the Kurucz (1979) model atmospheres using the $I U E$ spectra with the obviously underestimated $\log g=1.5$.

We can check these positions on the HR diagram, using the gravity values determined from our spectroscopic observations. The mean value is $\log g=2.35$, and it is very close to $\log g_{\text {puls }}$ for pulsation in the fundamental tone for a Cepheid with a pulsational mass of $2 M_{\odot}$ and a pulsational period of 1.95 , according to Wood et al. (1997). In case of the second overtone, the theoretical values are $\log g_{\text {puls }}=2.2, M_{\text {puls }}=3.0 M_{\odot}$ and $M_{\text {evol }}=$ 4.6 $M_{\odot}$. Using the HIPPARCOS distance $d=433 \mathrm{pc}$ and the absolute magnitude $M_{V}=-3$ m 25 we can obtain the $M_{\text {evol }}=5.2 M_{\odot}$ and $M_{\text {puls }}=3.7 M_{\odot}$.

Nevertheless, the uncertainty in $\log g=0.15$ dex would be a reason for some uncertainty in the pulsational mode identification. Furthermore, judging from Fig. 9, it is difficult to make a definite conclusion about it. The Cepheid's positions for the two values of luminosity in the HR diagram near the evolutionary tracks for $4 M_{\odot}$ and $5 M_{\odot}$ seems to be very uncertain. In short, the question about the pulsational mode of SU Cas remains open.

\section{Conclusions}

Based on the results of our detailed high-resolution spectroscopic investigation we can draw the following conclusions:

1) All studied Cas OB2 objects have metallicity values close to those of the Sun.

2) The mean $T_{\text {eff }}$ for SU Cas $(6325 \mathrm{~K})$ is the same as that $(6328 \mathrm{~K})$ determined by Turner \& Evans (1984) from $U B V$ - photometry and improved by accounting for the presence of a hot companion. The mean value of $\log g=2.35$ corresponds to pulsation in the fundamental tone, but the uncertainty in $\log g$ might suggest pulsation in an overtone. Nevertheless, the chemical abundance data for SU Cas show that the Cepheid is a post first dredge-up star. Hence, SU Cas is not crossing the instability strip for the first time. On the basis of these results and the positions of this Cepheid in the HR diagram for the two luminosity estimates, it is difficult to draw a conclusion regarding its evolutionary state and membership in the Cas OB2 association. Since overtone pulsations might suggest the first crossing of the Cepheid instability strip, the question about identification of the pulsational mode for SU Cas remains open. 
It would be interesting to estimate the age of SU Cas. From the Schaller et al. (1992) grids of models we can obtain the age values from $110^{8}\left(5 M_{\odot}\right)$ to $1.4510^{8}\left(4 M_{\odot}\right)$ years. These values are close to those found by Turner \& Evans (1984), $1.210^{8} \mathrm{yr}$.

3) Two stars of Cas OB2, HD 17327a and HD 16893, have approximately the same positions on the corresponding evolutionary tracks (see Fig. 9) and similar low rotational velocities. HD 17327a was discovered by us to be a $\mathrm{HgMn}$-star, which lies near the turn-off point of the association with the highest helium content among $\mathrm{HgMn}-$ stars and typical overabundance of $\mathrm{Mn}, \mathrm{Hg}, \mathrm{Sc}, \mathrm{Ti}, \mathrm{Cr}$, and $\mathrm{Y}$ and a deficit of $\mathrm{Al}, \mathrm{Mg}$ and $\mathrm{Si}$. This star is a primary component of a binary system with an uncertain orbital period. On the one hand, it is similar to the objects of the Searle-Sergeant group, while on the other hand, it is very similar to classical HgMn-stars. The overabundance of helium and other elements can be well explained by the LID mechanism in the stellar atmosphere. HD 16893 has an overabundance of $\mathrm{Mn}$, too, but it has a deficit of $\mathrm{C}, \mathrm{Mg}$ and $\mathrm{Si}$ with an overabundance of $\mathrm{Al}$ and $s$-process elements. Suggested as an Ap-type star (Turner \& Evans 1984), this object can be classified as an Am-star due to its manganese content. Additionally, it might have a strong magnetic field.

4) HD 17327b and HD 17443 are typical MS stars with high rotational velocities. Although HD 17443 is a more massive and more evolved object, it has a smaller $v \sin i$ value. It is remarkable that HD 17443 has a helium content, similar to that of the Sun.

Acknowledgements. Authors are thankful to Drs. D. G. Turner and A. Miroshnichenko for useful recommendations, to Dr. S. M. Andrievsky for help in the calculations and to Mrs A. Aret for discussion and information. Igor A. Usenko acknowledges support from the ISF (research grant YSU 082057).

\section{References}

Adelman, S. J., Philip, A. G. D., \& Adelman, C. J. 1996, MNRAS, 282, 953

Andrievsky, S. M., Kovtyukh, V. V., Usenko, I. A., Klochkova, V. G., \& Galazutdinov, G. A. 1996, A\&A, 305, 551

Atutov, S. N., \& Shalagin, A. M. 1988, SvA Lett., 14, 664

Barnes, T. G, Dominy, J. F., Evans, D. S., et al. 1977, MNRAS, 178,661

Batten, A. H., Fletcher, J. M., \& McCarthy, D. G. 1989, Publ. Dominion Astroph. Obs., 17, 1

Berdnikov, L. N., \& Pastukhova, E. N. 1994, SvA Lett., 20, 567

Castelli, F. 1991, A\&A, 251, 106

Chiosi, C., Wood, P. R., Bertelli, G., Bressan, A., \& Mateo, M. 1992, ApJ, 385, 205

Evans, N. R. 1991, ApJ, 372, 597

Evans, N. R. 1995, in Astrophysical Applications of Stellar Pulsations, ed. R. S. Stobie \& P. A. Whitelock, ASP Conf. Ser., 89, 252
Fernie, J. D., Beattie, B., Evans, N. R., \& Seager, S. 1993, IBVS, No. 4148

Galazutdinov, G. A. 1992, Preprint SAO RAS, No. 92

Gieren, W. P. 1982, PASP, 94, 960

Gieren, W. P., Fouque, P., \& Gomez, M. 1997, ApJ, 488, 74

Gorynya, N. A., Rastorguev, A. S., \& Samus, N. N. 1996, SvA Lett., 22, 38

Hubeny, I., Lanz, T., \& Jeffrey, C. S. 1994, Newsletter Analys. Astron. Spec., No. 20, 30

Kovtyukh, V. V., \& Andrievsky, S. M. 1999, A\&A, 351, 597

Kovtyukh, V. V., Gorlova, N. I., \& Klochkova, V. G. 1998, SvA Lett., 24, 372

Kurucz, R. L. 1979, ApJS, 40, 1

Kurucz, R. L., Furenlid, I., Brault, J., \& Testerman, L. 1984, The solar flux atlas from $299 \mathrm{~nm}$ to $1300 \mathrm{~nm}$, Nat. Sol. Obs., USA

Kurucz, R. L. 1992, The Stellar Populations of Galaxies, ed. B. Barbuy, A. Renzini, IAU Symp., 149, 225

Kurucz, R. L. 1995, Laboratory and Astronomical High Resolution Spectra, ed. A. J. Sauval, R. Blomme, \& N. Grevesse, ASP Conf. Ser., 81, 595

Lebedev, V. S. 1987, Astroph. Issled. Izv. SAO, 25, 41, in Russian

Luck, R. E., \& Lambert, D. L. 1981, ApJ, 245, 1018

Moon, T. T., \& Dworetsky, M. M. 1985, MNRAS, 217, 305

Napiwotzki, R., Schönberner, D., \& Wenske, V. 1993, A\&A, 268,653

Niva, G. D., \& Schmidt, E. G. 1979, ApJ, 234, 245

Panchuk, V. E., Najdenov, I. D., Klochkova, V. G., et al. 1998, Bull. SAO RAS, 44, 127

Panchuk, V. E., Klochkova, V. G., Najdenov, I. D., et al. 1999, Preprint SAO RAS, No. 139

Pavlova, L. A., \& Rspaev, F. K. 1986, Astrofizika, 25, 461

Racine, R. 1968, AJ, 73, 588

Ryabchikova, T. A. 1997, The Chemical Composition of Binary Stars with Mercury-Manganise Primary Components., in Binary Stars, ed. INASAN, Moscow, 105, in Russian

Ryabchikova, T. A., Zakharova, L. A., \& Adelman, S. J. 1996, MNRAS, 283, 1115

Schmidt, E. G. 1978, AJ, 83, 960

Searle, L., \& Sargent, W. L. W. 1967, The Magnetic and Related Stars (Baltimore: Mono Book Corp), 219

Schaller, G., Schaere, D., Meynet, G., \& Maeder, A. 1992, A\&AS, 96, 269

Stift, M. I. 1982, A\&A, 112, 149

Strajzys, V. 1982, in Metal-Deficient Stars, ed. Mokslas, Vilnius, 298

Szabados, L. 1991, Bud. Mitt. 11, No. 96, 125

Szabados, L. 1992, in Complementary Approaches to Double and Multiple Star Research, Proc. IAU Coll. 135, ed. H. A. McAlister, \& W. I. Hartkopf, ASP Conf. Ser. 32, 358

Szabados, L. 1997, in Proceed. of the ESA Symp. HIPPARCOS - Venice" 97", 13-16 May, Venice, Italy, ESA SP-402, 657

Takeda, Y., Kawanomoto, S., \& Ando, H. 1997, PASJ, 49, 493

Turner, D. G., \& Evans, N. R. 1984, ApJ, 283, 254

Usenko, I. A. 1990, Kinemat. i Fizika Nebesn. Tel. 6, No. 3, 91

Welch, D. L., \& Duric, N. 1988, AJ, 95, 1794

Wood, P., Capitanio, N., \& Chiosi, C. 1997, ApJS, 86, 541 